\title{
TRIVIAL MAXIMAL 1-ORTHOGONAL SUBCATEGORIES FOR AUSLANDER 1-GORENSTEIN ALGEBRAS
}

\author{
ZHAOYONG HUANG ${ }^{\bowtie}$ and XIAOJIN ZHANG
}

(Received 25 February 2011; accepted 8 June 2012; first published online 28 February 2013)

\author{
Communicated by D. Chan
}

\begin{abstract}
Let $\Lambda$ be an Auslander 1-Gorenstein Artinian algebra with global dimension two. If $\Lambda$ admits a trivial maximal 1-orthogonal subcategory of $\bmod \Lambda$, then, for any indecomposable module $M \in \bmod \Lambda$, the projective dimension of $M$ is equal to one if and only if its injective dimension is also equal to one, and $M$ is injective if the projective dimension of $M$ is equal to two. In this case, we further get that $\Lambda$ is a tilted algebra.
\end{abstract}

2010 Mathematics subject classification: primary 16G10; secondary 16E10.

Keywords and phrases: trivial maximal 1-orthogonal subcategories, Auslander 1-Gorenstein algebras, tilted algebras, indecomposable modules, projective dimension, injective dimension.

\section{Introduction}

Throughout this paper, $\Lambda$ is an Artinian algebra. We denote by $\mathbb{D}$ the ordinary duality, that is, $\mathbb{D}=\operatorname{Hom}_{R}\left(, I^{0}(R / J(R))\right)$, where $R$ is the center of $\Lambda, J(R)$ is the Jacobson radical of $R$ and $I^{0}(R / J(R))$ is the injective envelope of $R / J(R)$. We use mod $\Lambda$ and gl.dim $\Lambda$ to denote the category of finitely generated left $\Lambda$-modules and the global dimension of $\Lambda$, respectively.

Let $\mathscr{C}$ be a full subcategory of $\bmod \Lambda, M \in \bmod \Lambda$ and $C \in \mathscr{C}$. Recall from [4] that the homomorphism $f: M \rightarrow C$ is said to be a left $\mathscr{C}$-approximation of $M$ if $\operatorname{Hom}_{\Lambda}\left(C, C^{\prime}\right) \rightarrow \operatorname{Hom}_{\Lambda}\left(M, C^{\prime}\right) \rightarrow 0$ is exact for any $C^{\prime} \in \mathscr{C}$; the subcategory $\mathscr{C}$ is said to be covariantly finite in $\bmod \Lambda$ if every module in $\bmod \Lambda$ has a left $\mathscr{C}$-approximation. The notions of right $\mathscr{C}$-approximations and contravariantly finite subcategories of

This research was partially supported by the Specialized Research Fund for the Doctoral Program of Higher Education (Grant No. 20100091110034), NSFC (Grant Nos. 11171142, 11101217), NSF of Jiangsu Province of China (Grant Nos. BK2010047, BK2010007), the Fundamental Research Funds for the Central Universities (Grant No. 1116020304), NSF for Colleges and Universities in Jiangsu Province of China (Grant No. 11KJB110007), and a Project Funded by the Priority Academic Program Development of Jiangsu Higher Education Institutions.

(C) 2013 Australian Mathematical Publishing Association Inc. 1446-7887/2013 \$16.00 
$\bmod \Lambda$ may be defined dually. The subcategory $\mathscr{C}$ is said to be functorially finite in $\bmod \Lambda$ if it is both covariantly finite and contravariantly finite in $\bmod \Lambda$. For a positive integer $n$, we denote by ${ }^{\perp_{n}} \mathscr{C}=\left\{X \in \bmod \Lambda \mid \operatorname{Ext}_{\Lambda}^{i}(X, C)=0\right.$ for any $C \in \mathscr{C}$ and $1 \leq i \leq n\}$, and $\mathscr{C}^{\perp_{n}}=\left\{X \in \bmod \Lambda \mid \operatorname{Ext}_{\Lambda}^{i}(C, X)=0\right.$ for any $C \in \mathscr{C}$ and $\left.1 \leq i \leq n\right\}$. For a module $M \in \bmod \Lambda$, we use $\operatorname{add}_{\Lambda} M$ to denote the subcategory of $\bmod \Lambda$ consisting of all modules isomorphic to direct summands of finite direct sums of copies of ${ }_{\Lambda} M$.

One of the highlights in the representation theory of associative algebras is the theory of algebras of finite representation type. Following the fundamental work of Auslander and Reiten on the homological theory of almost split sequences [3], Gabriel, Bongartz and Riedtmann initiated the combinatorial approach to the representation theory based on Auslander-Reiten quivers [7, 11, 23]. In particular, Igusa, Todorov and Brenner gave beautiful combinatorial characterizations of Auslander-Reiten quivers of algebras of finite representation type [8, 16]. This theory also has an aspect as the structure theory of Auslander algebras, which are algebras of global dimension and dominant dimension two; actually Auslander algebras correspond bijectively to algebras of finite representation type [1]. They form a special class of noncommutative regular/Gorenstein rings studied by Auslander and Reiten [5, 6], and this interrelation of the representation theory and the homological algebra makes the whole theory very fruitful.

Recently Iyama introduced in [18] the notion of maximal $n$-orthogonal subcategories as the domain of higher analogue of the Auslander-Reiten theory. Recall that for a positive integer $n$, a functorially finite subcategory $\mathscr{C}$ of $\bmod \Lambda$ is called a maximal $n$-orthogonal subcategory if $\mathscr{C}={ }^{\perp_{n}} \mathscr{C}=\mathscr{C}^{\perp_{n}}$. Maximal $n$-orthogonal subcategories are called $(n+1)$-cluster tilting subcategories following Keller and Reiten's terminology in [21]. It is not difficult to see that any maximal $n$-orthogonal subcategory of $\bmod \Lambda$ contains $\operatorname{add}_{\Lambda}\left(\Lambda \oplus \mathbb{D} \Lambda^{\mathrm{op}}\right)$; and if $\operatorname{add}_{\Lambda}\left(\Lambda \oplus \mathbb{D} \Lambda^{\mathrm{op}}\right)$ is a maximal $n$-orthogonal subcategory of $\bmod \Lambda$, then $\operatorname{add}_{\Lambda}\left(\Lambda \oplus \mathbb{D} \Lambda^{\mathrm{op}}\right)$ is the unique maximal $n$ orthogonal subcategory of $\bmod \Lambda$. In this case, we say that $\Lambda$ admits a trivial maximal n-orthogonal subcategory of $\bmod \Lambda$ [14].

In general, maximal $n$-orthogonal subcategories rarely exist. So it is an important and interesting problem to investigate when maximal $n$-orthogonal subcategories (or modules) exist. This problem has been studied by several authors in $[9,12,14$, $15,18-20]$, for example. Iyama proved in [20] that if $\Lambda$ is a finite-dimensional algebra of finite representation type with Auslander algebra $\Gamma$ and $\bmod \Gamma$ contains a maximal 1-orthogonal module, then $\Lambda$ is hereditary and the injective envelope of ${ }_{\Lambda} \Lambda$ is projective; furthermore, if the base field is algebraically closed, then such $\Lambda$ is an upper triangular matrix ring. On the other hand, we gave in [14, Corollary 3.10] a necessary and sufficient condition for judging when an Auslander algebra admits a trivial maximal 1-orthogonal subcategory in terms of the homological dimensions of simple modules as follows. For an Auslander algebra $\Lambda$ with global dimension two, $\Lambda$ admits a trivial maximal 1-orthogonal subcategory of $\bmod \Lambda$ if and only if any simple module $S \in \bmod \Lambda$ with projective dimension two is injective. This result played an important role in studying the properties of Auslander algebras admitting 
a trivial maximal 1-orthogonal subcategory. For example, by applying it, we showed in [15, Corollary 4.11] the following result. Let $\Lambda$ be an Auslander algebra with global dimension two. If $\Lambda$ admits a trivial maximal 1-orthogonal subcategory of $\bmod \Lambda$, then $\Lambda$ is a tilted algebra of finite representation type. We also gave in [15] an example to illustrate that the conditions ' $\Lambda$ is an Auslander algebra' and ' $\Lambda$ is a tilted algebra of finite representation type' in this result cannot be exchanged. In this paper, we study the existence of trivial maximal 1-orthogonal subcategories under a weaker condition than above, and prove the following result.

THEOREM. Let $\Lambda$ be an Auslander 1-Gorenstein Artinian algebra with global dimension two. If $\Lambda$ admits a trivial maximal 1-orthogonal subcategory of $\bmod \Lambda$, then, for any indecomposable module $M \in \bmod \Lambda$ :

(1) the projective dimension of $M$ is equal to one if and only if its injective dimension is also equal to one;

(2) $M$ is injective if the projective dimension of $M$ is equal to two.

Furthermore, $\Lambda$ is a tilted algebra.

Recall that an Artinian algebra $\Lambda$ is called Auslander 1-Gorenstein if the injective envelope of ${ }_{\Lambda} \Lambda$ is projective. It was proved in [10, Theorem 3.7] that the notion of Auslander 1-Gorenstein algebras is left and right symmetric, that is, $\Lambda$ is Auslander $1-G o r e n s t e i n$ if and only if so is $\Lambda^{\mathrm{op}}$. Also recall that an Artinian algebra $\Lambda$ is called an Auslander algebra if the global dimension of $\Lambda$ is at most two and the first two terms in a minimal injective resolution of $\Lambda \Lambda$ are projective. So it is trivial that an Auslander algebra is Auslander 1-Gorenstein.

This paper is organized as follows. In Section 2 we give some notions and notation and collect some preliminary results. In Section 3 we study the homological properties of simple modules and indecomposable modules over an Artinian algebra with global dimension two and admitting a trivial maximal 1-orthogonal subcategory. As an application of the properties obtained, we prove the above theorem. At the end of this section, we give an example to illustrate the theorem.

\section{Preliminaries}

Let $M \in \bmod \Lambda$. We use $\operatorname{pd}_{\Lambda} M$ and $\operatorname{id}_{\Lambda} M$ to denote the projective and injective dimensions of $M$, respectively. We use

$$
\cdots \rightarrow P_{i}(M) \rightarrow \cdots \rightarrow P_{1}(M) \rightarrow P_{0}(M) \rightarrow M \rightarrow 0
$$

and

$$
0 \rightarrow M \rightarrow I^{0}(M) \rightarrow I^{1}(M) \rightarrow \cdots \rightarrow I^{i}(M) \rightarrow \cdots
$$

to denote a minimal projective resolution and a minimal injective resolution of $M$, respectively. Then $P_{0}(M)$ and $I^{0}(M)$ are a projective cover and an injective envelope of $M$, respectively. 
Lemma 2.1 [17, Proposition 1(3)]. For a nonnegative integer $n$, if $\operatorname{id}_{\Lambda} \Lambda=\operatorname{id}_{\Lambda^{\mathrm{op}}} \Lambda=n$, then $\mathrm{pd}_{\Lambda} E=n$ for any nonzero direct summand $E$ of $I^{n}(\Lambda)$.

We write $(-)^{*}$ for $\operatorname{Hom}_{\Lambda}(-, \Lambda)$.

Lemma 2.2 [22, Proposition 4.2]. Assume that $\operatorname{gl} \cdot \operatorname{dim} \Lambda=n(\geq 1)$. Then for any $0 \neq$ $M \in \bmod \Lambda$, the following statements are equivalent.

(1) $M$ has no nonzero projective direct summands and $\operatorname{Ext}_{\Lambda}^{i}(M, \Lambda)=0$ for any $1 \leq i \leq n-1$.

(2) By applying the functor $(-)^{*}$ to a minimal projective resolution of $M$, the induced sequence

$$
P_{0}(M)^{*} \rightarrow P_{1}(M)^{*} \rightarrow \cdots \rightarrow P_{n}(M)^{*} \rightarrow \operatorname{Ext}_{\Lambda}^{n}(M, \Lambda) \rightarrow 0
$$

is a minimal projective resolution of $\operatorname{Ext}_{\Lambda}^{n}(M, \Lambda)$ in $\bmod \Lambda^{\mathrm{op}}$.

Definition 2.3 [4]. A homomorphism $f: M \rightarrow N$ in $\bmod \Lambda$ is said to be left minimal if an endomorphism $g: N \rightarrow N$ is an automorphism whenever $f=g f$. The notion of right minimal homomorphisms is defined dually.

The following two properties of minimal homomorphisms are useful in the rest of the paper.

Lemma 2.4 [2, Ch. II, Lemma 4.3]. Let $0 \rightarrow A \stackrel{g}{\rightarrow} B \stackrel{f}{\rightarrow} C \rightarrow 0$ be a nonsplit exact sequence in $\bmod \Lambda$.

(1) If $C$ is indecomposable, then $g: A \rightarrow B$ is left minimal.

(2) If $A$ is indecomposable, then $f: B \rightarrow C$ is right minimal.

Lemma 2.5 [15, Lemma 2.5]. Let $0 \rightarrow A \stackrel{g}{\rightarrow} B \stackrel{f}{\rightarrow} C \rightarrow 0$ be a nonsplit exact sequence in $\bmod \Lambda$.

(1) If $g$ is left minimal, then $\operatorname{Ext}_{\Lambda}^{1}\left(C^{\prime}, A\right) \neq 0$ for any nonzero direct summand $C^{\prime}$ of $C$.

(2) If $f$ is right minimal, then $\operatorname{Ext}_{\Lambda}^{1}\left(C, A^{\prime}\right) \neq 0$ for any nonzero direct summand $A^{\prime}$ of $A$.

The following observation is easy to verify.

Lemma 2.6. Let $f: M \rightarrow N$ be a homomorphism in $\bmod \Lambda$.

(1) If $f$ is left minimal and $N=N_{1} \oplus N_{2}$ with $N_{1} \neq 0$, then $\left(1_{N_{1}}, 0\right) f \neq 0$.

(2) If $f$ is right minimal and $M=M_{1} \oplus M_{2}$ with $M_{1} \neq 0$, then $f\left(\begin{array}{c}1_{M_{1}} \\ 0\end{array}\right) \neq 0$.

The following useful lemma is due to T. Wakamatsu.

Lemma 2.7 [4, Lemma 1.3]. Let $\mathscr{C}$ be a full subcategory of $\bmod \Lambda$ which is closed under extensions and $D \in \bmod \Lambda$.

(1) If $D \stackrel{f}{\rightarrow} C \rightarrow Z \rightarrow 0$ is exact with $f$ a minimal left $\mathscr{C}$-approximation of $D$, then $\operatorname{Ext}_{\Lambda}^{1}(Z, \mathscr{C})=0$. 
(2) If $0 \rightarrow Z \rightarrow C \stackrel{f}{\rightarrow} D$ is exact with $f$ a minimal right $\mathscr{C}$-approximation of $D$, then $\operatorname{Ext}_{\Lambda}^{1}(\mathscr{C}, Z)=0$.

Definition 2.8 [18]. Let $\mathscr{C}$ be a functorially finite subcategory of $\bmod \Lambda$. For a positive integer $n, \mathscr{C}$ is called a maximal $n$-orthogonal subcategory of $\bmod \Lambda$ if $\mathscr{C}={ }^{\perp_{n}} \mathscr{C}=\mathscr{C}^{\perp_{n}}$.

From the definition above, it easily follows that both $\Lambda$ and $\mathbb{D} \Lambda^{\text {op }}$ are in any maximal $n$-orthogonal subcategory of $\bmod \Lambda$. Then $\operatorname{add}_{\Lambda}\left(\Lambda \oplus \mathbb{D} \Lambda^{\mathrm{op}}\right)$ is contained in any maximal $n$-orthogonal subcategory of $\bmod \Lambda$. On the other hand, it is easy to see that if $\operatorname{add}_{\Lambda}\left(\Lambda \oplus \mathbb{D} \Lambda^{\text {op }}\right)$ is a maximal $n$-orthogonal subcategory of $\bmod \Lambda$, then $\operatorname{add}_{\Lambda}\left(\Lambda \oplus \mathbb{D} \Lambda^{\mathrm{op}}\right)$ is the unique maximal $n$-orthogonal subcategory of $\bmod \Lambda$. In this case, we say that $\Lambda$ admits a trivial maximal n-orthogonal subcategory of $\bmod \Lambda$.

Lemma 2.9. Assume that $\operatorname{gl} \operatorname{dim} \Lambda=n(\geq 2)$ and $\Lambda$ admits a maximal $(n-1)$ orthogonal subcategory of $\bmod \Lambda$. Then:

(1) $I$ is projective or $\operatorname{pd}_{\Lambda} I=n$ for any injective module $I \in \bmod \Lambda$;

(2) $P$ is injective or $\operatorname{id}_{\Lambda} P=n$ for any projective module $P \in \bmod \Lambda$.

Proof. Because $\Lambda$ admits a maximal $(n-1)$-orthogonal subcategory of $\bmod \Lambda$, $\operatorname{Ext}_{\Lambda}^{i}(\mathbb{D} \Lambda, \Lambda)=0$ for any $1 \leq i \leq n-1$. For an injective module $I$ and a projective module $P$ in $\bmod \Lambda$, considering a minimal projective resolution of $I$ and a minimal injective resolution of $P$, respectively, then it is easy to obtain the assertions.

Let $\mathscr{C}$ be a full subcategory of $\bmod \Lambda$. We use $\mathscr{C}$ and $\overline{\mathscr{C}}$ to denote the stable subcategories of $\mathscr{C}$ modulo projective and injective modules, respectively. We use Ind $\mathscr{C}$ to denote the subcategory of $\mathscr{C}$ consisting of indecomposable modules in $\mathscr{C}$.

Lemma 2.10 [18, Theorem 2.2.4]. Assume that gl.dim $\Lambda=2$ and $\mathscr{C}$ is a maximal 1orthogonal subcategory of $\bmod \Lambda$. Then:

(1) there exist mutually inverse equivalences $\mathbb{D} \operatorname{Ext}_{\Lambda}^{2}(-, \Lambda): \underline{\mathscr{C}} \rightarrow \overline{\mathscr{C}}$ and $\operatorname{Ext}_{\Lambda}^{2}$ $(\mathbb{D}-, \Lambda): \overline{\mathscr{C}} \rightarrow \underline{\mathscr{C}}$

(2) $\mathbb{D} \operatorname{Ext}_{\Lambda}^{2}(-, \Lambda)$ gives a bijection from the nonprojective objects to the noninjective objects in Ind $\mathscr{C}$, and the inverse is given by $\operatorname{Ext}_{\Lambda}^{2}(\mathbb{D}-, \Lambda)$.

We recall the definition of almost hereditary algebras from [13].

Definition 2.11. $\Lambda$ is called an almost hereditary algebra if the following conditions are satisfied: (1) gl.dim $\Lambda \leq 2$; and (2) if $X \in \bmod \Lambda$ is indecomposable, then either $\operatorname{id}_{\Lambda} X \leq 1$ or $\operatorname{pd}_{\Lambda} X \leq 1$.

Also recall from [13] that $\Lambda$ is called a quasitilted algebra if $\Lambda=\operatorname{End}(T)$, where $T$ is a tilting object in a locally finite hereditary abelian $R$-category. It was proved in [13, Ch. III, Theorem 2.3] that $\Lambda$ is almost hereditary if and only if it is quasitilted, which implies that a tilted algebra is almost hereditary. On the other hand, it was shown in [14, Theorem 3.15] that if an almost hereditary algebra $\Lambda$ admits a maximal 1orthogonal subcategory $\mathscr{C}$ of $\bmod \Lambda$, then $\mathscr{C}$ is trivial (that is, $\mathscr{C}=\operatorname{add}_{\Lambda}\left(\Lambda \oplus \mathbb{D} \Lambda^{\mathrm{op}}\right)$ ). 
In the subcategory of mod $\Lambda$ consisting of indecomposable modules, we define the relation $\rightsquigarrow_{0}$ given by $X \rightsquigarrow_{0} Y$ if $\operatorname{Hom}_{\Lambda}(X, Y) \neq 0$, and let $\rightsquigarrow$ be the transitive closure of this relation, that is, $X \leadsto Y$ if and only if there exists a positive integer $n$ and a path $X=X_{0} \stackrel{f_{0}}{\rightarrow} X_{1} \stackrel{f_{1}}{\rightarrow} \cdots \rightarrow X_{n} \stackrel{f_{n}}{\rightarrow} X_{n+1}=Y$ of indecomposable modules $X_{i}$ and nonzero homomorphisms $f_{j}: X_{j} \rightarrow X_{j+1}$ for any $0 \leq i \leq n+1$ and $0 \leq j \leq n$. We use $\mathcal{R}_{\Lambda}$ to denote the subset of indecomposable $\Lambda$-modules given by $\mathcal{R}_{\Lambda}=\{X$ is indecomposable in $\bmod \Lambda \mid$ for all $X \leadsto Y$ we have $\left.\operatorname{id}_{\Lambda} Y \leq 1\right\}$ (see [13]).

Lemma 2.12. If $\Lambda$ is an Auslander 1-Gorenstein and almost hereditary algebra, then $\Lambda$ is a tilted algebra.

Proof. By the block theory of Artinian algebras, we can assume that $\Lambda$ is connected. Since $\Lambda$ is an almost hereditary algebra, all indecomposable injective $\Lambda$-modules belong to $\mathcal{R}_{\Lambda}$ by [13, Ch. II, Theorem 1.14]. Notice that $\Lambda$ is an Auslander 1-Gorenstein algebra, then there exists an indecomposable projective-injective module $I$ in $\bmod \Lambda$. So $I \in \mathcal{R}_{\Lambda}$. It follows from [13, Ch. II, Corollary 3.4] that $\Lambda$ is a tilted algebra.

\section{Homological dimensions of indecomposable modules}

In this section, we prove the main result by studying the homological properties of simple modules and indecomposable modules over an algebra with global dimension two and admitting a trivial maximal 1-orthogonal subcategory.

Throughout this section, assume that $\operatorname{gl} \cdot \operatorname{dim} \Lambda=2$ and $\Lambda$ admits a trivial maximal 1-orthogonal subcategory $\mathscr{C}\left(=\operatorname{add}_{\Lambda}\left(\Lambda \oplus \mathbb{D} \Lambda^{\mathrm{op}}\right)\right)$ of $\bmod \Lambda$. In this case, we then have that any indecomposable module in $\mathscr{C}$ is either projective or injective, and that $\operatorname{Ext}_{\Lambda}^{1}(I, P)=0$ for any injective module $I$ and any projective module $P$ in $\bmod \Lambda$. In the following, we will make free use of these facts.

The following lemma plays a crucial role in the proof of the main result in this paper.

Lemma 3.1. Let $S \in \bmod \Lambda$ be a noninjective simple module with $\operatorname{pd}_{\Lambda} S=2$. Then there exists a minimal right $\mathscr{C}$-approximation of $S$ :

$$
0 \rightarrow P \rightarrow I \rightarrow S \rightarrow 0
$$

satisfying the following conditions:

(1) $P$ is projective and $\operatorname{id}_{\Lambda} Q=2$ for any indecomposable direct summand $Q$ of $P$;

(2) I is injective and $\mathrm{pd}_{\Lambda} E=2$ for any indecomposable direct summand $E$ of $I$.

Proof. Because $\mathscr{C}\left(=\operatorname{add}_{\Lambda}\left(\Lambda \oplus \mathbb{D} \Lambda^{\text {op }}\right)\right)$ is a trivial maximal 1-orthogonal subcategory of $\bmod \Lambda, \mathscr{C}$ is functorially finite in $\bmod \Lambda$. Let $S \in \bmod \Lambda$ be a noninjective simple module with $\operatorname{pd}_{\Lambda} S=2$. Then there exists a minimal right $\mathscr{C}$-approximation of $S$ :

$$
0 \rightarrow P \rightarrow I \stackrel{f}{\rightarrow} S \rightarrow 0
$$


(1) By Lemma 2.7(2), $\operatorname{Ext}_{\Lambda}^{1}(\mathscr{C}, P)=0$ and $P \in \mathscr{C}^{\perp_{1}}(=\mathscr{C})$. So, by Lemma 2.5(2), for any indecomposable direct summand $Q$ of $P, Q$ is projective, but not injective. Then it follows from Lemma 2.9(2) that $\operatorname{id}_{\Lambda} Q=2$.

(2) Let $E$ be any indecomposable direct summand of $I$ such that $I=I^{\prime} \oplus E$. If $I^{\prime}=0$, then $E=I$ and $\operatorname{pd}_{\Lambda} E=2$. Now suppose that $I^{\prime} \neq 0$. By Lemma 2.6(2), we get the following commutative diagram with exact columns and rows:

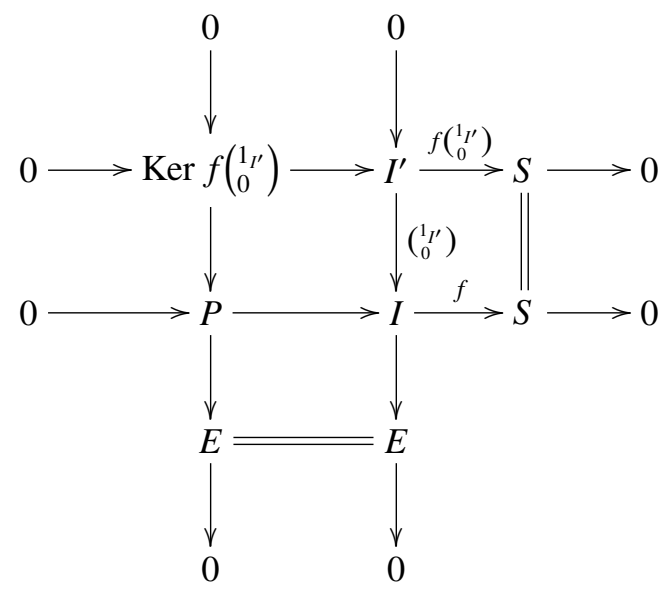

Note that $P$ is projective by (1). We claim that $E$ is not projective. Otherwise, if $E$ is projective, then $\operatorname{Ker} f\left(\begin{array}{l}1_{I^{\prime}} \\ 0\end{array}\right)$ is projective and the upper row in the above diagram is a right $\mathscr{C}$-approximation of $S$, which contradicts the fact that the middle row in the above diagram is a minimal right $\mathscr{C}$-approximation of $S$. The claim is proved. Then we conclude that $E$ is injective and $I$ is also injective. It follows from Lemma 2.9(1) that $\operatorname{pd}_{\Lambda} E=2$.

In the following, we will give some applications of Lemma 3.1, which are needed later. We first have the following lemma.

Lemma 3.2. $\operatorname{id}_{\Lambda} S \leq 1$ for any simple module $S \in \bmod \Lambda$ with $\operatorname{pd}_{\Lambda} S=2$.

Proof. If there exists a simple module $S \in \bmod \Lambda$ such that $\operatorname{pd}_{\Lambda} S=\mathrm{id}_{\Lambda} S=2$, then, by Lemma 3.1, there exists a minimal right $\mathscr{C}$-approximation of $S$ :

$$
0 \rightarrow P \stackrel{g}{\rightarrow} I \rightarrow S \rightarrow 0
$$

with $I$ injective. By Lemma 2.4(1), $g$ is left minimal. So

$$
0 \rightarrow P \stackrel{g}{\longrightarrow} I \rightarrow I^{0}(S) \rightarrow I^{1}(S) \rightarrow I^{2}(S) \rightarrow 0
$$

is a minimal injective resolution of $P$, and hence $\operatorname{id}_{\Lambda} P=3$, which contradicts the fact that gl.dim $\Lambda=2$.

The following result is another application of Lemma 3.1. 
Lemma 3.3. Let $S \in \bmod \Lambda$ be a simple module with $\operatorname{pd}_{\Lambda} S=2$ and $\operatorname{id}_{\Lambda} S=1$. Then:

(1) $\operatorname{Ext}_{\Lambda}^{1}(S, \Lambda) \in \bmod \Lambda^{\mathrm{op}}$ is projective;

(2) $\operatorname{Ext}_{\Lambda}^{2}(S, \Lambda) \in \bmod \Lambda^{\mathrm{op}}$ is injective and $\operatorname{pd}_{\Lambda^{\mathrm{op}}} \operatorname{Ext}_{\Lambda}^{2}(S, \Lambda)=2$.

Proof. Let $S \in \bmod \Lambda$ be a simple module with $\operatorname{pd}_{\Lambda} S=2$ and $\operatorname{id}_{\Lambda} S=1$. By Lemma 3.1, there exists a minimal right $\mathscr{C}$-approximation of $S$ :

$$
0 \rightarrow P \rightarrow I \rightarrow S \rightarrow 0
$$

such that $P(\neq 0)$ is projective, $I$ is injective without nonzero projective direct summands and $\operatorname{pd}_{\Lambda} I=2$.

By Lemma 2.2, $0 \rightarrow I^{*} \rightarrow P_{0}(I)^{*} \rightarrow P_{1}(I)^{*} \rightarrow P_{2}(I)^{*} \rightarrow \operatorname{Ext}_{\Lambda}^{2}(I, \Lambda) \rightarrow 0$ is a minimal projective resolution of $\operatorname{Ext}_{\Lambda}^{2}(I, \Lambda)$ in $\bmod \Lambda^{\mathrm{op}}$. Because gl.dim $\Lambda=2, I^{*}=0$. Then $\operatorname{Ext}_{\Lambda}^{1}(S, \Lambda) \cong P^{*}$ is projective. On the other hand, by Lemma 2.10, $\operatorname{Ext}_{\Lambda}^{2}(S, \Lambda) \cong$ $\operatorname{Ext}_{\Lambda}^{2}(I, \Lambda)$ is injective and $\operatorname{pd}_{\Lambda^{\text {op }}} \operatorname{Ext}_{\Lambda}^{2}(S, \Lambda)=2$.

As a consequence of Lemma 3.3 and the results obtained in Section 2, we have the following result.

Lemma 3.4. Let $S \in \bmod \Lambda$ be a simple module with $\operatorname{pd}_{\Lambda} S=2$ and $\operatorname{id}_{\Lambda} S=1$. Then

$$
0 \rightarrow \operatorname{Ext}_{\Lambda}^{1}(S, \Lambda) \rightarrow \operatorname{Ext}_{\Lambda}^{2}\left(I^{1}(S), \Lambda\right) \rightarrow \operatorname{Ext}_{\Lambda}^{2}\left(I^{0}(S), \Lambda\right) \rightarrow \operatorname{Ext}_{\Lambda}^{2}(S, \Lambda) \rightarrow 0
$$

is a minimal injective resolution of $\operatorname{Ext}_{\Lambda}^{1}(S, \Lambda)$ in $\bmod \Lambda^{\mathrm{op}}$.

Proof. Let $S \in \bmod \Lambda$ be a simple module with $\operatorname{pd}_{\Lambda} S=2$ and $\operatorname{id}_{\Lambda} S=1$. By applying the functor $(-)^{*}$ to a minimal injective resolution of $S$, we get an exact sequence in $\bmod \Lambda^{\mathrm{op}}$ :

$$
0 \rightarrow \operatorname{Ext}_{\Lambda}^{1}(S, \Lambda) \stackrel{\alpha}{\longrightarrow} \operatorname{Ext}_{\Lambda}^{2}\left(I^{1}(S), \Lambda\right) \rightarrow \operatorname{Ext}_{\Lambda}^{2}\left(I^{0}(S), \Lambda\right) \rightarrow \operatorname{Ext}_{\Lambda}^{2}(S, \Lambda) \rightarrow 0 .
$$

Because $S \in \bmod \Lambda$ is simple with $\operatorname{pd}_{\Lambda} S=2, \operatorname{Hom}_{\Lambda}\left(S, I^{2}(\Lambda)\right) \cong \operatorname{Ext}_{\Lambda}^{2}(S, \Lambda) \neq 0$ and $I^{0}(S)$ is isomorphic to a direct summand of $I^{2}(\Lambda)$. Because gl.dim $\Lambda=2$, $\operatorname{pd}_{\Lambda} I^{0}(S)=2$ by Lemma 2.1. Then $\operatorname{Ext}_{\Lambda}^{2}\left(I^{0}(S), \Lambda\right) \in \bmod \Lambda^{\text {op }}$ is indecomposable and injective by Lemma 2.10. On the other hand, $\operatorname{pd}_{\Lambda} I^{1}(S) \neq 1$ by Lemma 2.9, so $\operatorname{pd}_{\Lambda} I^{1}(S)=2$. In addition, by Lemma $2.5(1), I^{1}(S)$ has no nonzero projective direct summands. It follows from Lemma 2.10 that $\operatorname{Ext}_{\Lambda}^{2}\left(I^{1}(S), \Lambda\right) \in \bmod \Lambda^{\text {op }}$ is injective without nonzero projective direct summands.

By Lemma 3.3, $\operatorname{Ext}_{\Lambda}^{1}(S, \Lambda)$ is projective, so $\alpha$ is nonsplit. Because $\operatorname{Ext}_{\Lambda}^{2}\left(I^{0}(S), \Lambda\right)$ is indecomposable and injective, Coker $\alpha$ is indecomposable. Then, by Lemma 2.4(1), $\alpha$ is left minimal. Note that $\operatorname{Ext}_{\Lambda}^{2}(S, \Lambda) \in \bmod \Lambda^{\text {op }}$ is injective by Lemma 3.2(2). The proof is complete.

Now we can classify the simple modules over Auslander 1-Gorenstein algebras in terms of the projective and injective dimensions of simple modules as follows.

Proposition 3.5. Let $\Lambda$ be an Auslander 1-Gorenstein algebra and $S \in \bmod \Lambda$ a simple module. Then: 
(1) $S$ is injective if $\operatorname{pd}_{\Lambda} S=2$;

(2) $\operatorname{pd}_{\Lambda} S=1$ if and only if $\operatorname{id}_{\Lambda} S=1$.

Proof. (1) Let $S \in \bmod \Lambda$ be a simple module with $\operatorname{pd}_{\Lambda} S=2$. If $S$ is not injective, then, by Lemma 3.2, $\operatorname{id}_{\Lambda} S=1$. It follows from Lemmas 3.3(1) and 3.4 that

$$
0 \rightarrow \operatorname{Ext}_{\Lambda}^{1}(S, \Lambda) \rightarrow \operatorname{Ext}_{\Lambda}^{2}\left(I^{1}(S), \Lambda\right) \rightarrow \operatorname{Ext}_{\Lambda}^{2}\left(I^{0}(S), \Lambda\right) \rightarrow \operatorname{Ext}_{\Lambda}^{2}(S, \Lambda) \rightarrow 0
$$

is a minimal injective resolution of the projective module $\operatorname{Ext}_{\Lambda}^{1}(S, \Lambda)$ in $\bmod \Lambda^{\mathrm{op}}$. Because $\Lambda$ is Auslander 1-Gorenstein, $I^{0}\left(\Lambda^{\mathrm{op}}\right)$ is projective, which implies that $\operatorname{Ext}_{\Lambda}^{2}\left(I^{1}(S), \Lambda\right)$ is also projective. On the other hand, by Lemma 2.9, $\operatorname{pd}_{\Lambda} I^{1}(S) \neq 1$, so $\operatorname{pd}_{\Lambda} I^{1}(S)=2$ and hence $\operatorname{pd}_{\Lambda^{\text {op }}} \operatorname{Ext}_{\Lambda}^{2}\left(I^{1}(S), \Lambda\right)=2$ by Lemma 2.10, which is a contradiction.

(2) If $\operatorname{id}_{\Lambda} S=1$, then $\operatorname{pd}_{\Lambda} S \neq 2$ by (1) and $S$ is not projective by Lemma 2.9. So $\operatorname{pd}_{\Lambda} S=1$. Conversely, if $\operatorname{pd}_{\Lambda} S=1$, then $\operatorname{id}_{\Lambda^{\text {op }}} \mathbb{D} S=1$. Note that $\Lambda^{\text {op }}$ is also Auslander 1-Gorenstein and admits a trivial maximal 1-orthogonal subcategory of $\bmod \Lambda^{\mathrm{op}}$. By an argument dual to that above, $\operatorname{pd}_{\Lambda^{\text {op }}} \mathbb{D} S=1$ and $\operatorname{id}_{\Lambda} S=1$.

To prove the main result, we also need the following lemma.

Lemma 3.6. Let $\Lambda$ be an Auslander 1-Gorenstein algebra. If both $M$ and $P$ are indecomposable modules in $\bmod \Lambda$ such that $M$ is nonprojective and $P$ is projective with $\operatorname{id}_{\Lambda} P=2$, then $\operatorname{Hom}_{\Lambda}(M, P)=0$.

Proof. Let both $M$ and $P$ be indecomposable modules in $\bmod \Lambda$ such that $M$ is nonprojective and $P$ is projective with $\operatorname{id}_{\Lambda} P=2$. If there exists a nonzero homomorphism $f \in \operatorname{Hom}_{\Lambda}(M, P)$, then $f$ is not epic. Since $P$ is indecomposable and projective, $J(\Lambda) P$ is the unique maximal submodule of $P$ (where $J(\Lambda)$ is the Jacobson radical of $\Lambda)$ and $P / J(\Lambda) P(=S)$ is a simple module. Consider the following diagram:

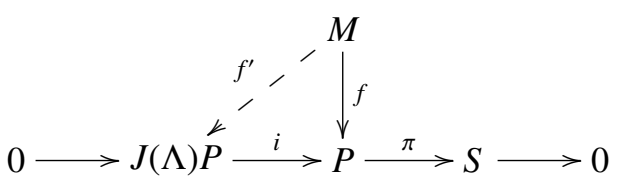

where $i$ is the inclusion homomorphism and $\pi$ is the natural epimorphism (which is a projective cover of $S$ ). Because $f(\neq 0)$ is not epic, there exists a nonzero homomorphism $f^{\prime}$ such that $f=i f^{\prime}$.

If $S$ is projective, then $P(\cong S)$ is simple and so $f$ is epic, which is a contradiction. If $\operatorname{pd}_{\Lambda} S=2$, then, by Proposition 3.5, $S$ is injective and $\mathbb{D} S$ is projective. On the other hand, $\mathbb{D} \pi: \mathbb{D} S \hookrightarrow \mathbb{D} P$ is an injective envelope of $\mathbb{D} S$ and $\operatorname{pd}_{\Lambda^{\mathrm{op}}} \mathbb{D} P=2$, which is also a contradiction because $\Lambda$ (and hence $\Lambda^{\mathrm{op}}$ ) is Auslander 1-Gorenstein. Thus we conclude that $\operatorname{pd}_{\Lambda} S=1$. So $J(\Lambda) P$ is projective and hence the injective dimension of any indecomposable direct summand of $J(\Lambda) P$ is equal to 2 by Lemmas 2.5(2) and 2.9. Because $f^{\prime} \neq 0$, there exists a nonzero indecomposable direct summand $P_{1}$ of 
$J(\Lambda) P$ such that $\operatorname{Hom}_{\Lambda}\left(M, P_{1}\right) \neq 0$. Then iterating the above process, we get an infinite descending chain of nonzero submodules of $P$,

$$
P \supsetneqq P_{1} \supsetneqq P_{2} \supsetneqq \cdots,
$$

which is a contradiction. The proof is complete.

We are now in a position to state the main result in this paper.

Theorem 3.7. Let $\Lambda$ be an Auslander 1-Gorenstein algebra. Then, for any indecomposable module $M \in \bmod \Lambda$ :

(1) $\operatorname{pd}_{\Lambda} M=1$ if and only if $\operatorname{id}_{\Lambda} M=1$;

(2) $M$ is injective if $\operatorname{pd}_{\Lambda} M=2$.

Proof. Let $M \in \bmod \Lambda$ be an indecomposable and nonprojective module. We claim that $\operatorname{id}_{\Lambda} M \leq 1$. Take a minimal left $\mathscr{C}$-approximation of $M$ :

$$
0 \rightarrow M \rightarrow C_{0} \rightarrow C_{1} \rightarrow 0
$$

If $P$ is a nonzero indecomposable and projective direct summand of $C_{0}$, then, by Lemma 2.6(1), $\operatorname{Hom}_{\Lambda}(M, P) \neq 0$. So $\operatorname{id}_{\Lambda} P \neq 2$ by Lemma 3.6, and hence $P$ is injective by Lemma 2.9. It follows that $C_{0}$ is injective.

If $C_{1}=0$, then $M \cong C_{0}$ is injective (in this case, $\operatorname{pd}_{\Lambda} M=2$ by Lemma 2.9). Now suppose that $C_{1} \neq 0$. By Lemma 2.7(1), $C_{1} \in{ }^{\perp_{1}} \mathscr{C}(=\mathscr{C})$. On the other hand, $C_{1}$ has no nonzero projective direct summands by Lemma $2.5(1)$, so $C_{1}$ is injective and hence $\operatorname{id}_{\Lambda} M=1$. The claim is proved.

(1) If $\operatorname{pd}_{\Lambda} M=1$, then $\operatorname{id}_{\Lambda} M=1$ by the above argument and Lemma 2.9. Because $\Lambda^{\mathrm{op}}$ is also Auslander 1-Gorenstein and admits a trivial maximal 1-orthogonal subcategory of $\bmod \Lambda^{\mathrm{op}}$, we get dually that $\mathrm{id}_{\Lambda} M=1 \mathrm{implies} \operatorname{pd}_{\Lambda} M=1$.

(2) By the above argument, it is easy to see that $M$ is injective if $\operatorname{pd}_{\Lambda} M=2$.

As an immediate consequence of Theorem 3.7, we get the following result.

Theorem 3.8. If $\Lambda$ is an Auslander 1-Gorenstein algebra, then $\Lambda$ is a tilted algebra.

Proof. If $\Lambda$ is an Auslander 1-Gorenstein algebra, then $\Lambda$ is almost hereditary by Theorem 3.7. It follows from Lemma 2.12 that $\Lambda$ is tilted.

To conclude this paper, we give the following example to illustrate the results obtained above.

ExAMPLE 3.9. Let $n \geq 4$ and $\Lambda$ be a finite-dimensional algebra given by the quiver

$$
1<\frac{\alpha_{1}}{\longleftarrow} 2<{ }^{\alpha_{2}}-3<\stackrel{\alpha_{3}}{\longleftarrow} \cdots \gtrless^{\alpha_{n-1}} n
$$

modulo the ideal generated by $\left\{\alpha_{1} \alpha_{2} \cdots \alpha_{n-1}\right\}$. Then: 
(1) $\Lambda$ is a tilted algebra of finite representation type, and admits a trivial maximal 1-orthogonal subcategory $\operatorname{add}_{\Lambda}\left[\left(\oplus_{i=1}^{n} P(i)\right) \oplus\left(\oplus_{i=3}^{n} I(i)\right)\right]$ of $\bmod \Lambda$. Because $I^{0}(\Lambda)$ is projective and $\operatorname{pd}_{\Lambda} I^{1}(\Lambda)=2, \Lambda$ is Auslander 1-Gorenstein, but not an Auslander algebra;

(2) for any indecomposable module $M \in \bmod \Lambda, \operatorname{pd}_{\Lambda} M=1$ if and only if id $M=1$, and $M$ is injective if $\operatorname{pd}_{\Lambda} M=2$.

\section{References}

[1] M. Auslander, Reprensentation Dimension of Artin Algebras, Queen Mary College Math. Notes (Queen Mary College, London, 1971).

[2] M. Auslander, 'Functors and morphisms determined by objects', in: Representation Theory of Algebras, Proc. Conf. Temple Univ. (Philadelphia, PA, 1976), Lecture Notes in Pure and Applied Mathematics, 37 (ed. R. Gordon) (Dekker, New York, 1978), 1-244.

[3] M. Auslander and I. Reiten, 'Representation theory of Artin algebras III: Almost split sequences', Comm. Algebra 3 (1975), 239-294.

[4] M. Auslander and I. Reiten, 'Applications of contravariantly finite subcategories', Adv. Math. 86 (1991), 111-152.

[5] M. Auslander and I. Reiten, ' $k$-Gorenstein algebras and syzygy modules', J. Pure Appl. Algebra 92 (1994), 1-27.

[6] M. Auslander and I. Reiten, 'Syzygy modules for noetherian rings', J. Algebra 183 (1996), 167-185.

[7] K. Bongartz and P. Gabriel, 'Covering spaces in representation-theory', Invent. Math. 65 (1981/82), 331-378.

[8] S. Brenner, 'A combinatorial characterization of finite Auslander-Reiten quivers', in: Representation Theory I: Finite Dimensional Algebras (Ottawa, Ont., 1984), Lecture Notes in Mathematics, 1177 (Springer, Berlin, 1986), 13-49.

[9] K. Erdmann and T. Holm, 'Maximal $n$-orthogonal modules for selfinjective algebras', Proc. Amer. Math. Soc. 136 (2008), 3069-3078.

[10] R. M. Fossum, P. A. Griffith and I. Reiten, Trivial Extensions of Abelian Categories, Lecture Notes in Mathematics, 456 (Springer, Berlin, 1975).

[11] P. Gabriel, 'The universal cover of a representation-finite algebra', in: Representations of Algebras (Puebla, 1980), Lecture Notes in Mathematics, 903 (Springer, Berlin, 1981), 68-105.

[12] C. Geiss, B. Leclerc and J. Schröer, 'Rigid modules over preprojective algebras', Invent. Math. 165 (2006), 589-632.

[13] D. Happel, I. Reiten and S. O. Smal, Tilting in Abelian categories and Quasitilted Algebras, Memoirs of the American Mathematical Society, 575 (American Mathematical Society, Providence, RI, 1996).

[14] Z. Y. Huang and X. J. Zhang, 'The existence of maxmal $n$-orthogonal subcateries', J. Algebra 321 (2009), 2829-2842.

[15] Z. Y. Huang and X. J. Zhang, 'Higher Auslander algebras admitting trivial maximal orthogonal subcategories', J. Algebra 330 (2011), 375-387.

[16] K. Igusa and G. Todorov, 'A characterization of finite Auslander-Reiten quivers', J. Algebra 89 (1984), 148-177.

[17] Y. Iwanaga and H. Sato, 'On Auslander's n-Gorenstein rings', J. Pure Appl. Algebra 106 (1996), 61-76.

[18] O. Iyama, 'Higher-dimensional Auslander-Reiten theory on maximal orthogonal subcategories', Adv. Math. 210 (2007), 22-50.

[19] O. Iyama, 'Auslander correspondence', Adv. Math. 210 (2007), 51-82.

[20] O. Iyama, 'Cluster tilting for higher Auslander algebras', Adv. Math. 226 (2011), 1-61. 
[21] B. Keller and I. Reiten, 'Cluster-tilted algebras are Gorenstein and stably Calabi-Yau', Adv. Math. 211 (2007), 123-151.

[22] Y. Miyashita, 'Tilting modules of finite projective dimension', Math. Z. 193 (1986), 113-146.

[23] Chr. Riedtmann, 'Algebren, Darstellungsköcher, Überlagerungen, und zurück', Comment. Math. Helv. 55 (1980), 199-224.

ZHAOYONG HUANG, Department of Mathematics, Nanjing University, Nanjing 210093, Jiangsu Province, PR China

e-mail: huangzy@nju.edu.cn

XIAOJIN ZHANG, College of Mathematics and Physics,

Nanjing University of Information Science and Technology, Nanjing 210044, Jiangsu Province, PR China

e-mail: xjzhang@nuist.edu.cn 